International Journal of Engineering \& Technology, $7(2.24)(2018) 259-262$
International Journal of Engineering \& Technology
WPC
Website: www.sciencepubco.com/index.php/IJET
Research paper

\title{
Multilevel Security System Using Raspberry Pi3
}

\author{
Karthikeyan. M*, Gowrishankar. KA, Rajith Sekar. G, E. Elamaran, B. Sudhakar \\ SRM Institute of Science and Technology, ${ }^{5}$ Annamalai University \\ *Corresponding Author Email: karthikeyanmuthu97@ gmail.com
}

\begin{abstract}
In this modern world security has become a major concern in securing a particular area such as banks, institution, temple premises etc. The existing system can be easily broken either by hacking the password or by duplicating entry cards. There are some security system which are very difficult to break but they are neither cost efficient nor easily affordable. We proposed a strong secure and affordable system using RFID, PIN lock and face recognition using raspberry pi3.
\end{abstract}

\section{Introduction}

In this project we propose a strong, reliable and affordable security system by integrating three system into a single system. The three systems are PIN lock system, RFID system, Face recognition system. The integrating component of all the three system is raspberry pi 3 . In the present situation the above three system act as a security system individually. In this paper we propose a system that combine all the three system.

\section{Objective}

The objective is to provide perfect high level security and to make the work easier, this project is taking help of different technologies viz. RFID, PIN lock, Face recognition using raspberry pi 3.

\section{Proposed System}

The proposed system consist of three blocks

- Face recognition block

- RFID block

- PIN system block

\section{A. Pin Lock Block}

The objective of this block is to prohibit unauthorized access by creating a PIN lock system that opens only when the authorized PIN of the users is entered. The PIN lock is supported by raspberry pi3 and LCD. When an authenticated PIN is entered the system will make way for RFID system if not the system will reject the user.

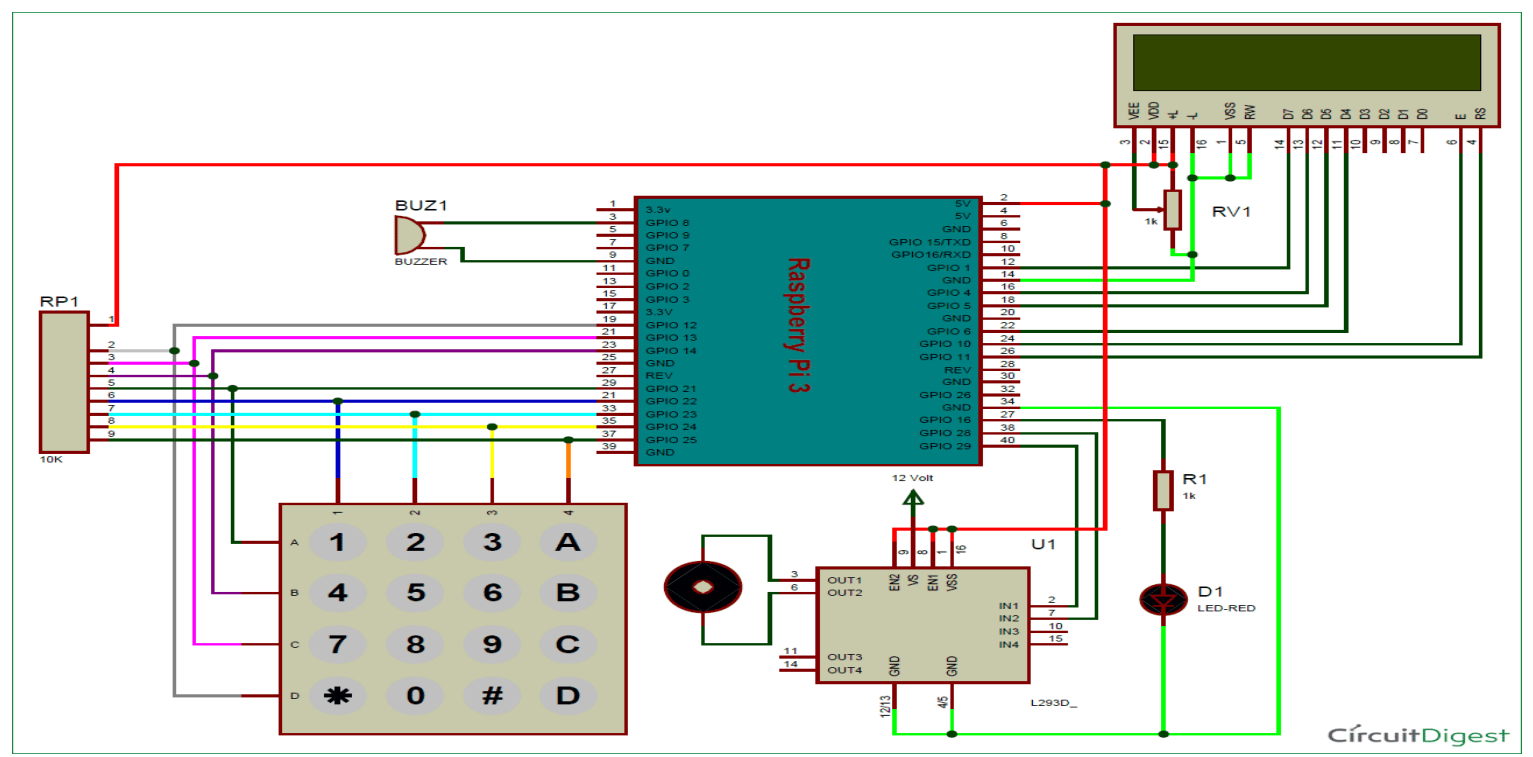

\section{B. RFID Block}

We are using RFID Reader Module that is connected through raspberry pi 3 , its basic use is to provide authorize access as this module can only provide access when you are having a access card if there is any unauthorized people try to break the security system them this would make active other parts of the system. The components of RFID block consist of RFID reader and RFID tag. 


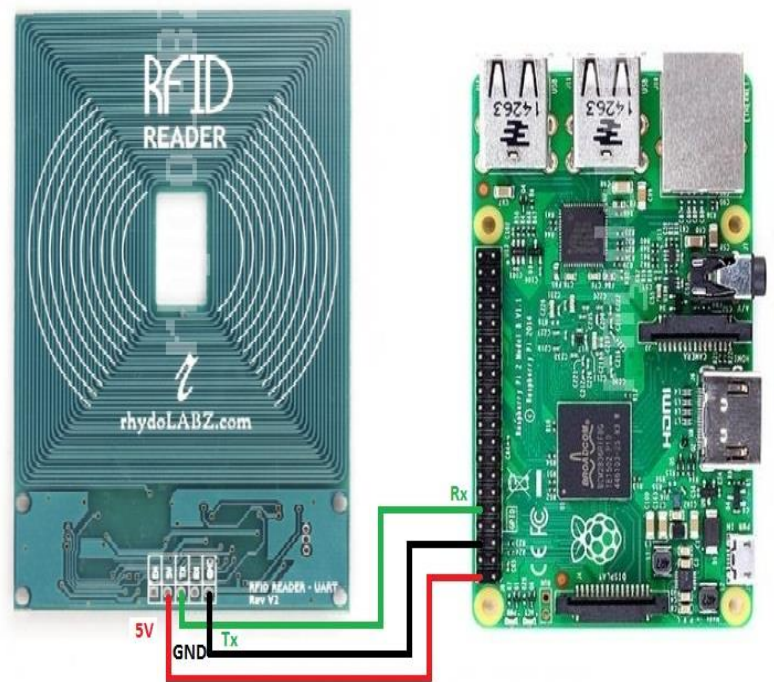

\section{1.) RFID Reader}

RFID Tag reader is used to read the RFID tag of the authenticated user. It works in the range of $100 \mathrm{KHz}-125 \mathrm{KHz}$ Its specifications are

- It is easy to interface.

-The supply voltage is between $7 \mathrm{~V}$ to $15 \mathrm{~V} \mathrm{DC}$.

- The operating voltage is between $4.5 \mathrm{~V}$ to $5 \mathrm{~V}$ DC.

- The operating frequency is between $100 \mathrm{KHz}$ to $125 \mathrm{KHz}$.

- It can read upto a distance of $5-10 \mathrm{~cm}$

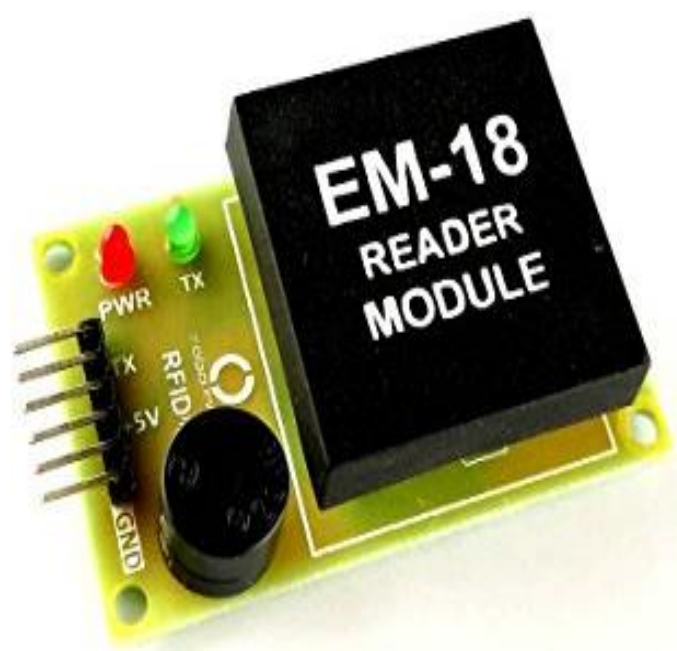

\section{2.) RFID Tag}

RFID tag in clam shell card type. 125Khz EM4001 compatible. Works with RFID reader modules RKI-1512 and RKI-1513. Range about $8-10 \mathrm{~cm}$. Its specifications are

- $125 \mathrm{kHz}$ read frequency and $10 \mathrm{~cm}$ range.

- EM4001 64-bit RFID tag compatible.

- 9600bps TTL and RS232 output.

- Magnetic stripe emulation output.

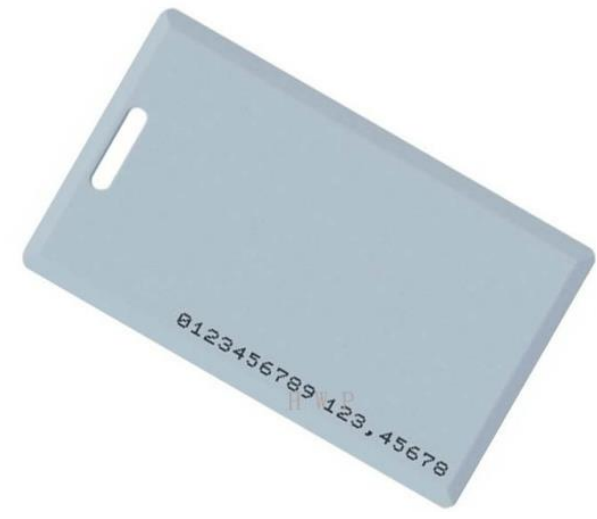

\section{Face Recognition Block}

This block maintains smart surveillance system using Raspberry Pi3, GSM module and Raspberry Pi-Camera. The camera keeps on recording the surrounding, Whenever a person comes in front of camera it checks with its database using local binary pattern algorithm. If the face matches with the ones in the database no alert is generated else host gets a text message on his android mobile phone via telegram application using WIFI adapter and GSM module, it also sends an email to the authentic user through WIFI. In this way, this system helps to identify only unauthorized persons. This helps to overcome the drawback of CCTV and Motion Detection systems which only monitor or alert host based on the motion detected whether it is authorized person or not. This block consist of Raspberry pi 3, camera, GSM module.

\section{1.) Raspberry PI 3}

It is a series of small single board computers used in various applications. It has several USB adapters, Ethernet port, WIFI adapter. It has a SD card slot in which about 32 GB memory space can be created. In this project it supports the face recognition block and integrating the other two systems into a single system.

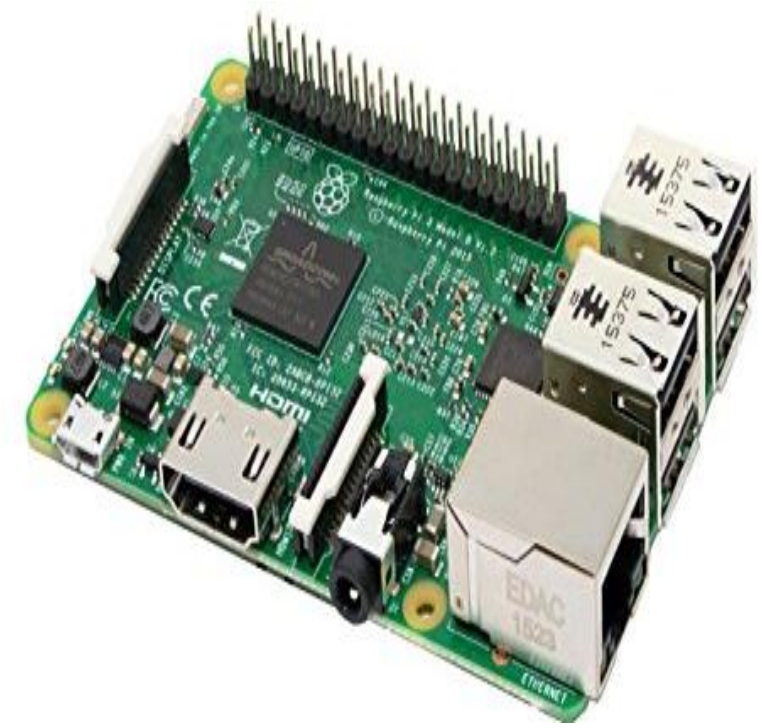

2.) Camera

A camera is an efficient remote sensing device used for image capturing and recording video. The camera can be wired or wireless and it captures the images or record a video without any contact with sensing object. The camera stores the recorded image or video in its allocated storage or it can be transferred to other devices. In this project the camera is connected with the raspberry pi 3 and it keeps on recording the surrounding. 


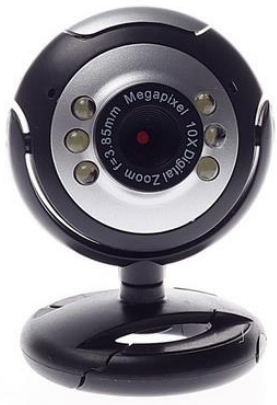

\section{3.) GSM Module}

GSM(Global System for mobile) is used for communication. It creates a path of communication between microprocessor and other system. In this project it is integrated with raspberry pi 3. It consist of a SIM slot through which the SMS will be sent to the authenticated user when an unauthorized person tries to access the system.

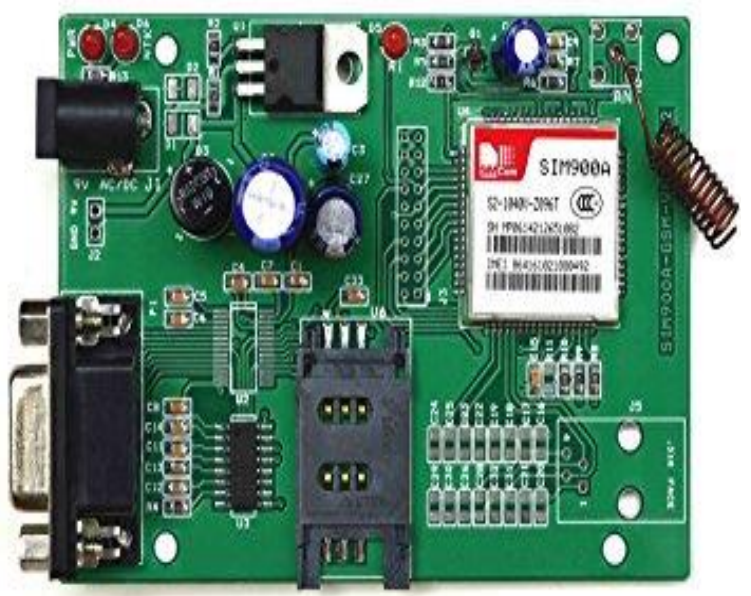

\section{Working}

- When an unauthorized person tries to access the system the access is prohibited by creating a PIN lock system that opens only when the authorized PIN of the users is entered.The PIN system is supported by LCD display. If the PIN lock matches, the user needs to place the RFID tag near the RFID reader. The door opens after the RFID reads the authorized RFID tag and displays the name of the authorized person in the LCD screen.

- The face is constantly monitored by camera controlled by raspberry pi3, if any unauthorized person is detected by the camera a text message with the name of the user's RFID card misused will be immediately sent to the user's mobile via GSM module and a small video is sent to the authorized person via telegram application via WIFI adapter in Raspberry PI3.

- The door opens after the access is granted by all the systems. Thus, a High Level security is achieved using the three levels of security.

- The three system makes this security system more strong, secure and the system respond in a very fast manner.

\section{Advantages}

- The advantage of the RFID system is that it works withoutline-of-sight.

- The communication between the user and the system is fast and reliable so that when an unauthorized person tries to enter the system, the user is immediately communicated by the system via SMS and a video via telegram application .
- The use of raspberry pi 3 makes the system easy to access and enables the user to change the features and function of the system in very simple and secure way.

- $\quad$ By using face recognition we can easily eliminate unauthorized user.

- The system is both reliable and cost efficient. It can be afforded by all sections of people.

\section{Block Diagram}

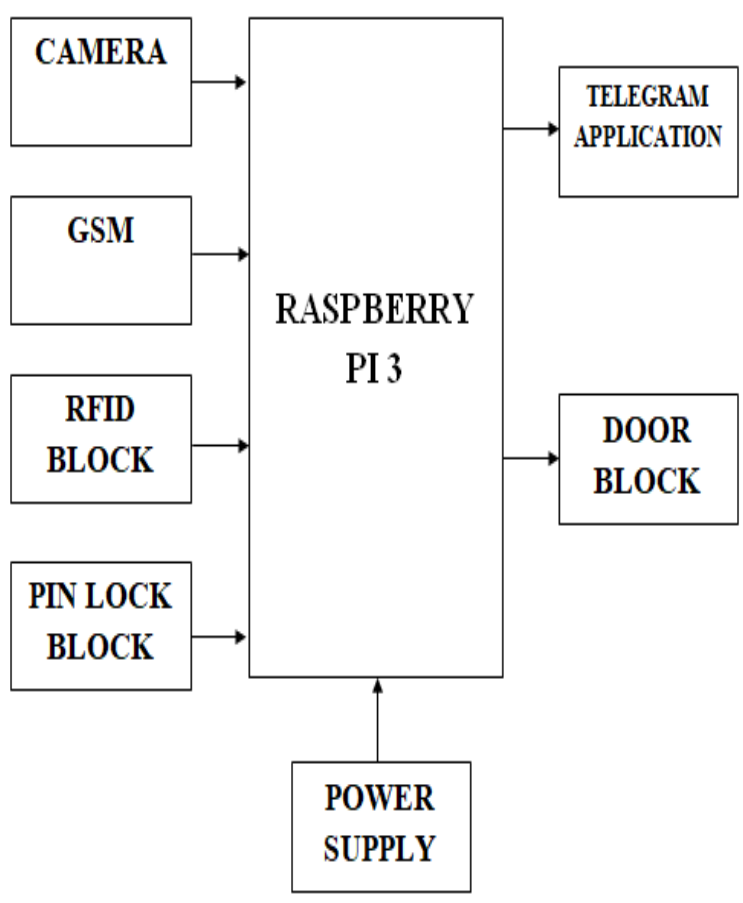

\section{Hardware and Software Description Hardware Description}

The proposed model aims at providing strong security system . In this model we have PIN lock, RFID block, Face recognition block. The PIN lock system is supported by LCD and raspberry pi 3. In the RFID block, it is also supported by raspberry pi 3, the RFID reader reads the RFID tag of each authenticated user. The LCD display in the RFID block show the user's name when they use their RFID tag. The raspberry pi3 controls the above two block along with the face recognition block. The last block face recognition block consist of GSM module along with SIM of the authenticated user. It is also supported by camera etc. The relay is used open the door system when all the three blocks authenticates the user.

\section{Software Description}

In this Project we are using multilevel security authentication system. To execute this system the coding part is more important. We use python programming console. All the three blocks are supported by raspberry pi 3supported by python program. In face recognition block the face is recognized with the input given with the help of python program. The all the three blocks is coordinated by raspberry pi3.

\section{Conclusion}

In the current work we developed a system by integrating three systems into a single system with the help of Raspberry pi 3. This projects paves way to build a cheap, strong and reliable security system for future research works. 


\section{Acknowledgement}

The work is being mainly done at SRM institute of science and technology, Kattankulathur, Chennai. We would like to thank our teachers and faculty of our college for their guidance for the progression in this project, they encouraged us to write this research paper. We are also grateful to our family for putting up with us.

\section{References}

[1] Ms. Naga Jyoti and Mr. K. Vijaya Vardhan, "Design And Implementation Of Real Time Security Surveillance System Using IoT". Communication and Electronics Systems (ICCES), International Conference: IEEE, 2016.

[2] RFID BASED SECURITY AND ACCESS CONTROL SYSTEM USING ARDUINO WITH GSM MODULE Grewal Kaushal, Rishabh Mishra, Neelam Chaurasiya, Paramdeep Singh Vol. 2, Issue 2 (April, 2015).

[3] Smart Surveillance System using Raspberry $\mathrm{Pi}$ and Face Recognition Vol. 6, Issue 4, April 2017

[4] Aamir Nizam Ansari, Mohamed Sedky, Neelam Sharma, Anurag Tyagi, "An Internet of Things Approach for Motion Detection using Raspberry Pi", International Conference on Intelligent Computing and Internet of Things (IC1T), 2015. 